IMPACTO DEL MICROCRÉDITO EN LAS CONDICIONES DE VIDA (INGRESO Y CULTURA EMPRESARIAL) DE MUJERES TABASQUEÑAS. CASO COMPARTAMOS BANCO.

\title{
IMPACTO DEL MICROCRÉDITO EN LAS CONDICIONES DE VIDA (INGRESO Y CULTURA EMPRESARIAL) DE MUJERES TABASQUEÑAS. CASO COMPARTAMOS BANCO.
}

\section{THE IMPACT OF MICROCREDIT IN TABASQUEAN WOMEN'S LIFE CONDITIONS (INCOME AND COMPANY CULTURE). COMPARTAMOS BANCO STUDY CASE.}

\author{
Roberto Alfonso Cordero Olivares*
}

*Maestría en Análisis y Gestión Financiera. Universidad Autónoma de Guadalajara, Campus Tabasco. ORCID: https://orcid.org/0000-0003-4800-0838.

Dirección para recibir correspondencia: raco1250@hotmail.com 
IMPACTO DEL MICROCRÉDITO EN LAS CONDICIONES DE VIDA (INGRESO Y CULTURA EMPRESARIAL) DE MUJERES TABASQUEÑAS. CASO COMPARTAMOS BANCO.

\section{RESUMEN}

En Tabasco, durante los últimos años se incrementó el número de sucursales de Compartamos Banco, al día de hoy operan más de 10 sucursales, que otorgan créditos a mujeres, con el objetivo de poner en marcha su propio negocio o ayudando a quienes ya cuentan con uno para mejorar su bienestar económico.

OBJETIVO: Analizar la experiencia de seis mujeres beneficiarias de microcréditos otorgados por Compartamos Banco en Villahermosa, Tabasco y su impacto en las condiciones de vida: ingresos y cultura empresarial a partir del otorgamiento del crédito.

MATERIAL Y MÉTODO: Investigación con diseño cualitativo. Se utilizó la técnica de entrevista a profundidad y los datos fueron analizados con el software Atlas Ti.

RESULTADOS: Las entrevistas muestran que las mujeres que recibieron el microcrédito no han logrado salir de la pobreza, mantienen deudas y no poseen conocimientos suficientes para alcanzar una rentabilidad real en sus emprendimientos.

CONCLUSIONES: El "Crédito Mujer" otorgado por Compartamos Banco a seis emprendedoras de Villahermosa, Tabasco, no es un instrumento efectivo en la reducción de la pobreza, por el contrario genera mayor endeudamiento y obliga a las mujeres a permanecer por largos periodos en grupos de préstamos.

PALABRAS CLAVES: Microfinanzas. Microcréditos. Mujeres tabasqueñas. Compartamos Banco.

\section{ABSTRACT}

In Tabasco, a number of branches of Compartamos Banco has increased over the years. Nowadays, 10 branches are operating in the state where credits are awarded to women, with the objective of setting up their own businesses or helping them to improve their wealth.

OBJECTIVE: To analyze the experience of six women who were granted with a microcredit by Compartamos Banco in Villahermosa, Tabasco; and the impact on life conditions: income and company culture from the credit grant. 
IMPACTO DEL MICROCRÉDITO EN LAS CONDICIONES DE VIDA (INGRESO Y CULTURA EMPRESARIAL) DE MUJERES TABASQUEÑAS. CASO COMPARTAMOS BANCO.

MATERIAL AND METHOD: Qualitative research. The in-depth interview techniques was used and the information was analyzed with the Atlas ti software.

RESULTS: Findings show that women who were granted with a microcredit are still in poverty, they still have debts and do not possess enough knowledge to reach a real profitability level in their start-ups.

CONCLUSIONS: The credit called "Credito Mujer" granted to six entrepreneur women by Compartamos Banco in Villahermosa, Tabasco it is not an effective instrument to reduce poverty, on the contrary, it generates a greater debt and forces women to remain for a longer period of time in loaning groups.

KEY WORDS. Microfinance. Microcredit. Tabasquean women. Compartamos Banco.

\section{INTRODUCCIÓN}

Las Instituciones Microfinancieras (IMF) se convirtieron en una de las herramientas favoritas para combatir la pobreza en los países en desarrollo (Castillo, 2008). El origen de este concepto surge a mediados de los 70's por el economista Muhammad Yunus fundador del Banco Grameen en Bangladesh. Este hecho dio un impulso a nivel mundial al movimiento de microcrédito, la práctica está vinculada con el movimiento cooperativo rural iniciado por W.F. Raiffeisen y promovida por los bancos Raiffeisen y el Volksbanken de Alemania, el Rabobank de Holanda y el Crédit Agricole de Francia (Kumar, Hossain, \& Gope, 2015).

El acelerado crecimiento de los microfinanciamientos, se puede explicar por cuatro factores básicos: 1) Enorme demanda de servicios financieros por millones de personas de bajos ingresos, 2) Tecnología para proveer servicios a la población, 3) Inversionistas y agencias dispuestos a invertir y 4) Difusión mundial. De acuerdo con datos del Programa de las Naciones Unidas para el Desarrollo (PNUD, 2016), a nivel mundial, alrededor de 736 millones de personas viven con menos de US\$1.90 al día, muchos carecen de acceso alimentos, agua potable y saneamiento. Siendo las mujeres quienes se encuentran en mayor desventaja social debido al acceso desigual al trabajo remunerado, la educación y la propiedad. 
IMPACTO DEL MICROCRÉDITO EN LAS CONDICIONES DE VIDA (INGRESO Y CULTURA EMPRESARIAL) DE MUJERES TABASQUEÑAS. CASO COMPARTAMOS BANCO.

\section{MARCO TEÓRICO}

En 2004, la Organización de Naciones Unidas (ONU) declaró el 2005 como el Año Internacional del Microcrédito, con el objetivo de promoverlo como una estrategia contra la pobreza (ONU, 2004). La ONU instó a los gobiernos a generar programas de microcrédito para disminuir la pobreza, de esta manera fomentar el trabajo por cuenta propia y generar ingresos (Aguilar-Pinto, Tuñón-Pablos \& Morales-Barragán (2017).

Si bien, la iniciativa puede ayudar a revertir la situación de empobrecimiento, no incide en el problema estructural de la pobreza (Vega-Estrada, 2014). De acuerdo con el Consejo Nacional de la Política Social (Coneval, 2012) una persona es pobre, cuando tiene por lo menos uno de los seis indicadores de carencia social: 1) rezago educativo, 2) acceso a servicios de salud, 3) acceso a la seguridad social, 4) calidad y espacios de la vivienda, 5) servicios básicos en la vivienda y 6) acceso a la alimentación; y su ingreso es insuficiente para adquirir los bienes y servicios que requiere para satisfacer sus necesidades alimentarias y no alimentarias.

El 70\% de los pobres del mundo son mujeres, debido a lo cual desde siempre se han visto desfavorecidas en el acceso al crédito y a otros servicios financieros. Los bancos comerciales suelen centrarse en los hombres y las empresas formales, pasando por alto a las mujeres, que constituyen un enorme y creciente segmento de la economía informal.

El porcentaje de mujeres en situación de pobreza pasó de $44.6 \%$ a $42.4 \%$ entre 2008 y 2018, en tanto que el porcentaje de hombres en situación de pobreza fue de $44.0 \%$ a $41.4 \%$ entre 2008 y 2018 (Coneval, 2019).

De acuerdo con datos del Coneval (2016), los hogares dirigidos por mujeres tienden a contar con mayor carencia en acceso a la alimentación. Durante el 2012, la carencia ascendió a 41.5\%, contra $34.9 \%$ de los hogares encabezados por varones pobres. A pesar de contar con la misma escolaridad, las remuneraciones a mujeres por sus empleos son menores a las que perciben los hombres.

\section{Las microfinanzas como atenuantes de la pobreza femenina}

La investigación "Social Responsability of Microfinance: Banco Compartamos Case" concluye que es responsabilidad de las microfinancieras, favorecer la inclusión de las mujeres a créditos, 
IMPACTO DEL MICROCRÉDITO EN LAS CONDICIONES DE VIDA (INGRESO Y CULTURA EMPRESARIAL) DE MUJERES TABASQUEÑAS. CASO COMPARTAMOS BANCO.

acceso a educación financiera y desarrollo del espíritu emprendedor (Vargas-Hernández \& Santos Huerta, 2015).

De acuerdo con el Informe publicado en 2010 Fondo Multilateral de Inversiones, miembro del Grupo del Banco Interamericano de Desarrollo (BID) (FOMIN, 2010) las mujeres experimentan múltiples beneficios al ser sujetos de microcrédito como es el desarrollo de la propia identidad, el reconocimiento de sus capacidades y limitaciones, autonomía económica y el desarrollo de su autoestima, en la medida que son capaces de tomar decisiones importantes sobre ellas, sus familias y la economía.

Datos del Coneval (2019), muestran que el porcentaje de mujeres mexicanas en pobreza incrementó de 2008 a 2018 en un 6.1\%, principalmente en el sureste del país.

En Tabasco, durante los últimos años ha habido un crecimiento en el número de sucursales de Compartamos Banco, al día de hoy operan más de 10 sucursales, dispone de establecimientos que están ubicados dentro de las principales colonias de la ciudad de Villahermosa, estas sucursales tienen como propósito realizar el cobro de los créditos otorgados a los beneficiarios, además de convertirse en una red estructurada de promotores y supervisores, con lo que han logrado el posicionamiento de la empresa.

A pesar de existir un número elevado de empresas que otorgan créditos a mujeres, estas parecen no salir de la pobreza. Los créditos resultan un paliativo que no logra acabar con la desigualdad y mejorar de forma tangible las condiciones de vida de las mujeres.

Las teorías sobre las microfinanzas y microcréditos están cambiando, al afirmar que estos no pueden resolver todos los problemas que existen en el mundo, se necesitan políticas públicas más efectivas que ayuden a mitigar la pobreza, principalmente en la región sureste de México.

\section{Compartamos Banco "Crédito Mujer"}

Compartamos es el microprestamista más grande de México. Nace en 1990 como una Organización No Gubernamental (ONG). En 2006 se convierte en banco comercial, siendo público hasta el 2007. Actualmente, cuenta con más de 2.5 millones de clientes y maneja 6 productos, destacando el producto "Crédito Mujer", el cual representa aproximadamente el 80\% de su cartera total. Por representatividad y por ser considerado insignia del banco, es importante 
IMPACTO DEL MICROCRÉDITO EN LAS CONDICIONES DE VIDA (INGRESO Y CULTURA EMPRESARIAL) DE MUJERES TABASQUEÑAS. CASO COMPARTAMOS BANCO.

profundizar en el funcionamiento del producto "Crédito Mujer" objeto de estudio de la investigación.

Consiste en reunir grupos compuesto por un mínimo de 10 mujeres emprendedoras, en donde se otorga un crédito individual a cada una de ellas de acuerdo a la necesidad de su actividad económica. Los montos van desde los 5 mil hasta los 70 mil pesos. El $75 \%$ de la cartera de crédito se concentra en 26 actividades económicas, dentro de las que destacan la compra venta de ropa con un $25.3 \%$, tiendas de abarrotes y misceláneas con un $13.6 \%$ (Espinosa \& Gabilondo, 2008).

Los créditos tienen un plazo de vencimiento de 16 semanas. A pesar de ser un crédito individual, Compartamos Banco toma como aval solidario al grupo. Lo que implica un único crédito que se pagará semanalmente y que, por ende, estará conformado por la suma de todos los créditos individuales. Esta metodología implica la creación de un grupo solidario, el cual se compromete a pagar de manera semanal el pago pactado con el banco, independientemente de que uno de los miembros haya incumplido con su parte. Si el grupo tiene atrasos en los pagos, cae en cartera vencida y ya no es susceptible de renovación del crédito para el siguiente ciclo.

\section{OBJETIVO}

Analizar la experiencia de seis mujeres beneficiarias de créditos otorgados por Compartamos Banco en Villahermosa, Tabasco y su impacto en las condiciones de vida: ingresos y cultura empresarial a partir del otorgamiento del crédito.

\section{MATERIAL Y MÉTODO}

Se aplicó una entrevista online con 38 preguntas a un grupo de mujeres que fueron beneficiarias del "Crédito Mujer" otorgado por Compartamos Banco, con el propósito de crear un perfil sociodemográfico y empresarial de cada una de las participantes.

El estudio profundizó en temas como la composición familiar, con el propósito de descubrir cuál es el rol económico y personal que tienen cada una de ellas en la familia, conocer si son las proveedoras principales o aportan parte de sus ingresos a la economía familiar, si están casadas, solteras, tienen hijos, cuántas personas dependen económicamente de ellas. En el tema profesional era necesario conocer los niveles de escolaridad, capacitación previa en negocios, finanzas, creación de planes de negocios; por último, se indagó en el tema personal, autoconocimiento y autoestima. Era indispensable conocer si las mujeres que están CORDERO-OLIVARES R. A. 
IMPACTO DEL MICROCRÉDITO EN LAS CONDICIONES DE VIDA (INGRESO Y CULTURA EMPRESARIAL) DE MUJERES TABASQUEÑAS. CASO COMPARTAMOS BANCO.

emprendiendo han pasado por un proceso de autodescubrimiento, si conocen sus talentos, habilidades, conocimientos, su razón de ser y hacer. Se utilizó la aplicación google forms para obtener la información y los datos se procesaron con el software Atlas TI, para análisis de datos cualitativos.

\section{RESULTADOS}

Para los propósitos de la investigación, era necesario conocer algunos aspectos socioeconómicos, demográficos y culturales de las mujeres beneficiarias del "Crédito Mujer" otorgado por Compartamos Banco, con el objetivo de comprender y analizar los perfiles de cada una de ella y conocer el impacto que ha tenido este beneficio en su vida y si ha ayudado o no a superar sus limitaciones financieras, generar nuevas fuentes de empleo, mejorar su autoestima, tomar decisiones importantes.

De acuerdo con los resultados de la entrevista, el 75\% de las mujeres son solteras, tienen un empleo formal y además generan ingresos con sus negocios. Todas mencionaron que han emprendido algún negocio gracias a la ayuda de los créditos, que su situación económica actual es regular y que han tenido en algunas ocasiones dificultad para pagar sus créditos. El ingreso promedio de las mujeres entrevistadas oscila entre los 7 mil y 10 mil pesos mensuales, cinco de ellas renta, no tienen casa propia, manejan más de dos tarjetas de crédito y tienen dependientes económicos.

Un dato importante que arrojó la entrevista está relacionado con el "éxito de su emprendimiento actual", el $100 \%$ de las mujeres dijo sentirse poco satisfecha con los resultados actuales de su negocio. Las entrevistas mostraron que cinco de las mujeres que han recibido el "Crédito Mujer" otorgado por Banco Compartamos tienen un promedio de escolaridad de bachillerato, pocos conocimientos sobre marketing, finanzas personales, ventas, emprendimiento, gestión de proyectos, liderazgo y administración de empresas. No basta con tener una idea brillante y el financiamiento para realizar un emprendimiento, es necesario poseer conocimientos y saber cómo aplicarlos dentro de un mercado que cambia constantemente (Carrillo, Bergamini \& Navarro, 2014). Otro aspecto que era importante conocer es el giro comercial de los emprendimientos. En la figura 1, se muestra las actividades en las que las mujeres invierten el crédito. 
IMPACTO DEL MICROCRÉDITO EN LAS CONDICIONES DE VIDA (INGRESO Y CULTURA EMPRESARIAL) DE MUJERES TABASQUEÑAS. CASO COMPARTAMOS BANCO.

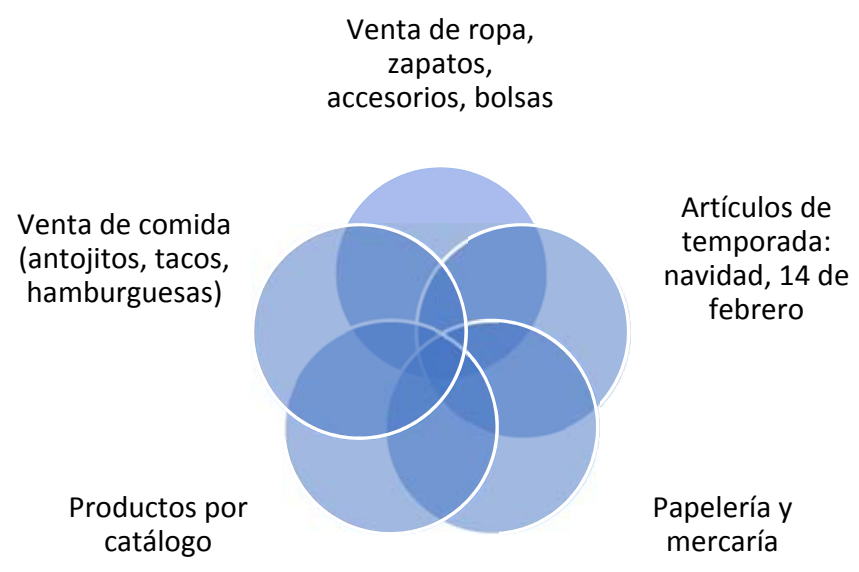

Figura 1. Giro comercial de los emprendimientos.

Fuente: Elaboración propia.

La segunda parte de la entrevista se enfoca en preguntar ¿Por qué ha solicitado un crédito? Algunas de las respuestas fueron: Para generar otras ganancias; otra respondió para superar crisis de pagos y rentas y comprar productos de venta; para tener dinero y comprar mercancías; iniciar emprendimiento y pagar deudas. Los mensajes de las mujeres evidencian que los créditos son utilizados principalmente para pagar deudas y comprar productos, en las entrevistas también se comentó que han utilizado el dinero del crédito para cubrir gastos personales y pagar otros créditos. Tal parece que los créditos no resuelven el problema de la pobreza, por el contrario genera un endeudamiento, por lo que las usuarias se ven en la necesidad de volver a utilizar un crédito de este tipo. La investigación de García-Horta \& Zapata-Martelo (2012), comprobó que los microcréditos no aseguran que los pequeños emprendimientos sean auto sostenibles económicamente.

Otra de las preguntas fue ¿Consideras que tu vida ha cambiado o mejorado gracias a los créditos de Compartamos Banco? Las mujeres respondieron: Sí, un poco; no mucho; en algunas ocasiones he salido de mis problemas gracias a un préstamo aunque después me sigo endeudando. Fueron las respuestas más reiteradas. Continuando con la entrevista se pidió una visión a futuro de su situación y su relación con los microcréditos, mediante la siguiente pregunta ¿En el futuro pediría otro crédito? Las respuestas fueron divididas, algunas de ellas fueron positivas y otras no. Si, Si para poner otro negocio; Ya no lo pediría, puesto que significa asumir deudas y la situación es precaria por el momento. 
IMPACTO DEL MICROCRÉDITO EN LAS CONDICIONES DE VIDA (INGRESO Y CULTURA EMPRESARIAL) DE MUJERES TABASQUEÑAS. CASO COMPARTAMOS BANCO.

Las respuestas reflejan que los microcréditos no han cambiado las condiciones de vida de las mujeres, ellas permanecen en las mismas condiciones e incluso como algunas manifiestan "la situación es precaria en este momento", por lo tanto no estaría dispuesta a pedir otro crédito. Esto obliga a reflexionar que las mujeres están utilizando los créditos como una alternativa para salir de problemas personales, pero no para seguir consolidando sus proyectos, hacerlos crecer, comprar nueva tecnología, contratar personal, llevar su negocio a un siguiente nivel, etc., Por ello, las usuarias realizan emprendimientos de actividades como: hacer comida, vender artículos por catálogo, venta de ropa, accesorios, bolsas, carteras, esto no quiere decir que las mujeres no posean el conocimiento o habilidades para desarrollarse en otro sector o generar emprendimientos de alto impacto. Lo que queda muy claro es, que otorgar microcréditos a emprendedoras no es una solución para disminuir la pobreza y generar mejores condiciones de vida a las mujeres.

\section{CONCLUSIONES}

La estrategia de los microcréditos y las microfinanzas, parte del supuesto de que las mujeres pobres necesitan capital para utilizarlo en actividades que les permitan superar las condiciones de pobreza. La investigación muestra que las mujeres con créditos de Compartamos Banco no han tenido un cambio significativo en sus condiciones económicas, su nivel de ingreso sigue siendo el mismo, además muchas de ellas no han logrado consolidar sus negocios, utilizan el crédito para otras actividades que tienen nulo impacto en el desarrollo de su emprendimiento. Por ejemplo, varias de ellas manifestaron que han utilizado el crédito para realizar pagos de deudas, resolver situaciones de tipo personal y algunas para comprar mercancía para mantener activo el negocio. Un dato que es importante analizar tiene que ver con el nivel educativo de las participantes y sus conocimientos sobre temas que son de vital importancia para un emprendedor.

La mayoría de las mujeres aceptó no poseer conocimientos en temas como: marketing, ventas, administración de empresas, finanzas, liderazgo, los cuales son necesarios para que un emprendedor pueda llevar al éxito su pequeño negocio. Es importante hacer énfasis en la formación de los usuarios de microcréditos para que hagan uso adecuado de los fondos, incluyendo entrenamiento en la puesta en marcha y administración de microempresas.

Las microfinanzas, por sí solas, no pueden contribuir a reducir la pobreza, aumentar el ingreso, incrementar activos económicos, mejorar la educación, disminuir la desigualdad social o la vulnerabilidad, o dar un mayor empoderamiento si todo ello no se acompaña de adecuados CORDERO-OLIVARES R. A. 
IMPACTO DEL MICROCRÉDITO EN LAS CONDICIONES DE VIDA (INGRESO Y CULTURA EMPRESARIAL) DE MUJERES TABASQUEÑAS. CASO COMPARTAMOS BANCO.

procesos que otorguen conocimiento, participación, acceso, financiación e intervención sobre las personas más vulnerables (Tuñón, Tinoco \& Hernández, 2007). Por ello, en colectivos pobres y sin acceso al crédito, el elemento que determina su situación de exclusión y privación es la falta de reconocimiento social e institucional para acceder a dispositivos y recursos públicos, no la falta de crédito, como con frecuencia se ha afirmado (Gómez, 2017).

Se ha sobrestimado el potencial de los microcréditos entre la población más vulnerable, debido a que han vendido la falsa idea que ayudan a reducir la pobreza, disminuir deuda e incluso generar riqueza, cuando la realidad es que lo único que están creando es sobreendeudamiento de los usuarios que no cuentan con la orientación adecuada de cómo administrar correctamente los recursos obtenidos (Riaño-Marín, \& Okali, 2008).

En la investigación ningún usuario manifestó tener ingresos superiores que le permitan vivir únicamente de su emprendimiento, por esa razón transitan entre la esfera de lo formal e informal. Se observa que esto crédito son solo paliativos que en poco ayudan a las mujeres a mejorar sus condiciones de vida. El tema es alarmante ya que lo que interesa a la empresa prestadora, es el pago del crédito y evitar que los usuarios caigan en cartera vencida. Por lo tanto, no sé está generando una verdadera transformación de la sociedad, ni contribuyendo a mejorar las condiciones de vida de las emprendedoras.

\section{REFERENCIAS BIBLIOGRÁFICAS}

Aguilar-Pinto, E.Tuñón-Pablos, E. \& Morales-Barragán, F. (2017). Microcrédito y pobreza. La experiencia del programa Microempresas Sociales de Banmujer en Chiapas. Economía, sociedad y territorio, 17(55), 809-835. Recuperado de http://www.scielo.org.mx/scielo.php?script=sci_arttext\&pid=S1405-84212017000300809

Carrillo, L., Bergamini, T. \& Navarro, C. (2014). El emprendimiento como motor del crecimiento económico. Boletín económico de ICE, Información Comercial Española, 3048, 55-63. Recuperado de: https://dialnet.unirioja.es/servlet/articulo?codigo=5253484

Castillo, A. (2008). El microcrédito como instrumento para el alivio de la pobreza: Ventajas y limitaciones. Cuadernos de Desarrollo rural, 5(61), 93-110. Recuperado de: https://www.redalyc.org/pdf/117/11713138004.pdf 
IMPACTO DEL MICROCRÉDITO EN LAS CONDICIONES DE VIDA (INGRESO Y CULTURA EMPRESARIAL) DE MUJERES TABASQUEÑAS. CASO COMPARTAMOS BANCO.

Consejo Nacional de Evaluación de la Política de Desarrollo Social. (Coneval). (2012), Pobreza

y género en México. Hacia un sistema de indicadores. Recuperado de: https://www.coneval.org.mx/Medicion/MP/Paginas/Pobreza-y-genero-en-México.aspx

Consejo Nacional de Evaluación de la Política de Desarrollo Social. (Coneval). (2016). Medición de la Pobreza 2008-2018. Recuperado de: https://www.coneval.org.mx/Medicion/MP/Paginas/Pobreza_2016.aspx

Consejo Nacional de Evaluación de la Política de Desarrollo Social. (Coneval). (2019).10 años de medición de pobreza en México, avances y retos en política social. Recuperado de: https://www.coneval.org.mx/SalaPrensa/Comunicadosprensa/Documents/2019/COMUNIC ADO_10_MEDICION_POBREZA_2008_2018.pdf

Espinosa, A \& Gabilondo, E. (2008). Valuación de una Institución de Banca Múltiple: Banco Compartamos (tesis de maestría). Instituto Tecnológico de Monterrey Campus Ciudad de México, México. Recuperado de: https://repositorio.tec.mx/handle/11285/632310

Fondo Multilateral de Inversiones (FOMIN). (2010). Dueña de tu propia empresa: Identificación, análisis y superación de las limitaciones a las pequeñas empresas de las mujeres en América Latina y el Caribe. Recuperado de https://publications.iadb.org/es/publicacion/13690/duena-de-tu-propia-empresaidentificacion-analisis-y-superacion-de-las

García-Horta, J. \& Zapata-Martelo, E. (2012). El papel de las microfinanzas en la pobreza y desigualdad de las mujeres. Ra Ximhai, 8(1), 101-111. Recuperado de: https://www.redalyc.org/pdf/461/46123324007.pdf

Gómez, C. (2017).El fenómeno de los microcréditos en la cooperación mundial: límites y controversias. $\quad$ E-DHC, 6-20. Recuperado de: https://rua.ua.es/dspace/bitstream/10045/93961/1/2019_Gomez-Gil_E-DHC.pdf

Kumar, D., Hossain, A., \& Gope, M. C. (2015). Role of micro credit program in empowering rural women in Bangladesh: A study on Grameen Bank Bangladesh Limited. Asian Business Review, 3(4), 114-120. Recuperado de: http://www.academia.edu/download/37290947/287-911-1-PB.pdf

Organización de las Naciones Unidas (ONU). (2004). Año Internacional del Microcrédito. Recuperado de https://www.un.org/es/events/pastevents/microcredit/ 
IMPACTO DEL MICROCRÉDITO EN LAS CONDICIONES DE VIDA (INGRESO Y CULTURA EMPRESARIAL) DE MUJERES TABASQUEÑAS. CASO COMPARTAMOS BANCO.

Programa de las Naciones Unidas para el Desarrollo (PNUD). (2016). Desde los ODS hasta el desarrollo sostenible para todos. Recuperado de: https://www.undp.org/content/dam/undp/library/SDGs/Spanish/ES_f_UNDP_MDGs-toSDGs_web.pdf

Riaño-Marín, R. E. \& Okali, C. (2008). Empoderamiento de las mujeres a través de su participación en proyectos productivos: experiencias no exitosas. Convergencia, 15(46), 119-141. Recuperado de http://www.scielo.org.mx/scielo.php?pid=S140514352008000100006\&script=sci_arttext\&tlng=pt

Tuñón, E., Tinoco, R. \& Hernández, A. (2008). Género y microfinanciación: evaluación de un programa de microcréditos para mujeres en el estado de Tabasco, México. La ventana. Revista de estudios de género, 3(26), 41-69. Recuperado de: http://www.scielo.org.mx/scielo.php?pid=S1405-

94362007000200041\&script=sci_abstract\&tIng=en

Vargas-Hernández, J. \& Santos Huerta, M. (2015). Social Responsibility of Microfinance: Banco Compartamos Case (Responsabilidad Social De Microfinancieras: El Caso Compartamos Banco). Available at SSRN 2561715 Recuperado de: https://papers.ssrn.com/sol3/papers.cfm?abstract_id=2533019

Vega-Estrada, S. (2014). Para contender con la pobreza. México: Porrúa. 